\section{Fusion of bone-marrow-derived cells with Purkinje neurons, cardiomyocytes and hepatocytes}

\section{Manuel Alvarez-Dolado ${ }^{1}$, Ricardo Pardal ${ }^{2}$, Jose M. Garcia-Verdugo ${ }^{3}$ John R. Fike ${ }^{1}$, Hyun O. Lee ${ }^{2}$, Klaus Pfeffer ${ }^{4}$, Carlos Lois ${ }^{5}$, Sean J. Morrison ${ }^{2}$ \& Arturo Alvarez-Buylla}

${ }^{1}$ Department of Neurological Surgery, University of California at San Francisco, San Francisco, California 94143-0520, USA

${ }^{2}$ Howard Hughes Medical Institute, Department of Internal Medicine, University of Michigan, Ann Arbor, Michigan 48109-0934, USA

${ }^{3}$ Instituto Cavanilles, University of Valencia, Valencia 46100, Spain

${ }^{4}$ Institute of Medical Microbiology, University of Dusseldorf, D-40225 Dusseldorf, Germany

${ }^{5}$ Picower Center for Learning and Memory, Massachusetts Institute of Technology, Cambridge, Massachusetts 02139-4307, USA

Recent studies have suggested that bone marrow cells possess a broad differentiation potential, being able to form new liver cells, cardiomyocytes and neurons ${ }^{1,2}$. Several groups have attributed this apparent plasticity to 'transdifferentiation' ${ }^{3-5}$. Others, however, have suggested that cell fusion could explain these results ${ }^{6-9}$. Using a simple method based on Cre/lox recombination to detect cell fusion events, we demonstrate that bone-marrow-derived cells (BMDCs) fuse spontaneously with neural progenitors in vitro. Furthermore, bone marrow transplantation demonstrates that BMDCs fuse in vivo with hepatocytes in liver, Purkinje neurons in the brain and cardiac muscle in the heart, resulting in the formation of multinucleated cells. No evidence of transdifferentiation without fusion was observed in these tissues. These observations provide the first in vivo evidence for cell fusion of BMDCs with neurons and cardiomyocytes, raising the possibility that cell fusion may contribute to the development or maintenance of these key cell types.

In order to detect cell fusion we used a method based on Cre/lox recombination, a technique extensively used to conditionally turn on or off gene expression in specific cell types or tissues, or at particular stages in development ${ }^{10}$. For this study we first used mice expressing Cre recombinase ubiquitously under the control of a hybrid cytomegalovirus (CMV) enhancer $\beta$-actin promoter ${ }^{11}$ (Fig. 1a), and the conditional Cre reporter mouse line $R 26 \mathrm{R}^{12}$ (Fig. 1b). In this line, the LacZ reporter gene is exclusively expressed after the excision of a loxP-flanked (floxed) stop cassette by Cremediated recombination (Fig. 1b). When Cre-expressing $\left(\mathrm{Cre}^{+}\right.$) cells fuse with R26R cells, Cre recombinase excises the floxed stop cassette of the reporter gene in the R26R nuclei, resulting in expression of LacZ in the fused cells. Consequently, fused cells can be detected easily by 5 -bromo-4-chloro-3-indolyl- $\beta$-D-galactoside (X-gal) staining (Fig. 1c). This method previously failed to detect evidence of cell fusion in the pancreas ${ }^{13}$. For this reason, we first verified this cell fusion detection method in vitro.

We co-cultured bone marrow stromal cells (BMSCs) from R26R reporter mice with $\mathrm{Cre}^{+}$multipotent progenitor cells isolated from postnatal brain and grown as neurospheres ${ }^{14}$. Previous studies have shown that these two cell types can fuse with embryonic stem cells in vitro $^{6,7}$. After 4 days in vitro (DIV), a small proportion of $\beta$-gal ${ }^{+}$ cells were found in these co-cultures ( 1 to 2 cells per 80,000 cells) (Fig. 1d). Importantly, most $\beta$-gal ${ }^{+}$cells at 4 DIV had two or more nuclei, an observation that was confirmed by electron microscopy (Fig. 1e, i; see also Supplementary Fig. 1). This is consistent with the generation of $\beta-$ gal $^{+}$cells by fusion. Notably, after 10 or 15 DIV $\beta$-gal ${ }^{+}$cells formed small colonies and some of these cells were mitotic (Fig. $1 \mathrm{~g}$ and inset). Cells in these colonies were invariably mononucleated, suggesting that with time and cell division the nuclei of these cells fuse or supernumerary nuclei are eliminated. In addition to neurosphere cells, R26R BMSCs were also co-cultured with primary cultures of $\mathrm{Cre}^{+}$fibroblasts. Three independent co-cultures did not yield positive cells, suggesting that not all cell types are equally capable of fusion in culture. To further confirm that $\beta$-gal expression was due to cell fusion, $\mathrm{Cre}^{+}$neurosphere cells were labelled with 5-bromodeoxyuridine (BrdU) and then cocultured for 5 days with R26R BMSCs. Most bi-nucleated $\beta$-gal ${ }^{+}$ cells in these cultures had only one of the two nuclei labelled with BrdU (Fig. 1h-k), further confirming the reliability of this method to detect fusion events.

These results confirm previous work demonstrating that cell fusion occurs spontaneously in vitro ${ }^{6,7}$. To study cell fusion in vivo, R26R reporter mice were lethally irradiated, and 2 days later were grafted with bone marrow from mice constitutively expressing Cre recombinase and green fluorescent protein (GFP) under the control of the $\beta$-actin promoter $(\beta$-actin-Cre-GFP mice, see 'allogeneic bone marrow transplantation' section of Methods). We analysed the grafted mice at $2(n=3)$ and $4(n=3)$ months after transplantation. These mice showed significant levels of haematopoietic reconstitution, which was measured by flow cytometry based on the frequency of $\mathrm{GFP}^{+}$cells in peripheral blood (from $54.6 \%$ to $79.8 \%$ of nucleated blood cells). Brain, liver, heart, gut, kidney, lung and skeletal muscle from these mice were serially sectioned and stained for the presence of $\mathrm{X}-\mathrm{gal}^{+}$cells. In all animals, cells labelled with $\beta$-gal were only found in brain, heart and liver, and not in the other organs studied (Table 1). As a negative control, we grafted R26R mice with bone marrow from wild-type mice. We did not find $\beta$-gal ${ }^{+}$cells in these animals, demonstrating that the reporter was not inappropriately activated, even after irradiation.

BMDCs fuse with hepatocytes in fumarylacetoacetate-hydrolasedeficient mice ${ }^{8,9}$. Consistent with this finding, we also observed fused hepatocytes in our grafted mice (Fig. 2). $\beta$-gal ${ }^{+}$hepatocytes expressed albumin (a characteristic hepatocyte marker) (Fig. 2, h) but were negative for CD45 (a haematopoietic marker) (data not shown). Electron microscopy confirmed that these fused cells were typical hepatocytes with no features of haematopoietic cells (Fig. 2c). They contained glycogen granules, complete desmosomes and bile canaliculi (Fig. 2d). Two months after transplant most $\beta$-gal ${ }^{+}$hepatocytes co-expressed the donor marker GFP (Fig. 2e, f); however, a small fraction of fused hepatocytes were GFP negative. This fraction had increased 4 months after transplantation (Table 1). This result suggests that after fusion donor genes may be inactivated/eliminated over time.

At 2 and 4 months after transplantation, $\beta$-gal ${ }^{+}$cells were detected in the cerebellum, where labelled cells displayed the typical location and morphology of Purkinje cells (Fig. 3a). Two $\beta$-gal ${ }^{+}$ cells were embedded in plastic and serially sectioned for light and electron microscopy. Serial reconstruction of the soma of these cells demonstrated the presence of two nuclei (Fig. 3b; see also Supplementary Fig. 2). Notably, the two nuclei presented very different morphologies: one had a wrinkled surface with multiple invaginations and a single nucleolus, typical of Purkinje cells ${ }^{15}$, whereas the second nucleus showed a uniform spherical shape with multiple nucleoli, suggesting a different origin (Fig. 3b, c). Electron microscopy analysis confirmed that these cells were Purkinje neurons (Fig. 3c) with typical Purkinje cell somata and organelle distribution, including structures with the characteristics of synaptic contacts (Fig. 3d). No signs of degeneration or abnormal structures were observed in the cytoplasm of these cells (Fig. 3c, d). $\beta$-gal ${ }^{+}$Purkinje cells stained positively for the Purkinje cell marker calbindin (data not shown). This is the first direct evidence, to our knowledge, showing that a neuron can fuse with a BMDC. These results suggest that previous observations of small numbers of Purkinje cells bearing markers of transplanted 
bone marrow cells ${ }^{5,16,17}$ may have arisen by fusion rather than transdifferentiation.

The third organ where $\beta$-gal ${ }^{+}$cells were found was the heart (Fig. 4). The $\beta$-gal ${ }^{+}$cells were integrated into the myocardial wall and had a morphology and alignment that was indistinguishable from the surrounding cardiac muscle fibres (Fig. 4a-d). At the electron microscopy level, the fused cells had the morphology of mature cardiomyocytes, including developed filament bands and mature intercalated discs with desmosomes and GAP junctions connecting to neighbouring fibres (Fig. 4e). In addition, fused cardiomyocytes expressed cardiac troponin I (Fig. 4g). As seen in the liver, GFP was expressed in most of the fused cardiomyocytes at 2 months after transplantation (Table 1). In contrast, fused cardiomyocytes lost GFP expression at 4 months (Fig. 4h). It has been suggested that haematopoietic stem cells can partially restore the infarcted heart by transdifferentiation, giving rise to new myocardium $^{4}$. Our results suggest that BMDCs can fuse with cells within the heart to form mature cardiomyocytes, but it remains unknown whether any new cardiomyocytes are generated as a result of this fusion.

Bone marrow cells include haematopoietic cells as well as mesenchymal cells and possibly other cell types. To test whether haematopoietic cells were participating in fusion events in vivo we used donor mice in which Cre recombinase was knocked into the CD45 locus (CD45-Cre). As CD45 is specifically expressed by haematopoietic cells ${ }^{18,19}$, recombination should only occur in fusions involving cells of the haematopoietic lineage. To confirm the CD45-Cre expression pattern these mice were mated with R26R reporter mice. We observed widespread $\beta$-gal expression in haematopoietic stem cells, bone marrow cells, and blood cells but not in other tissues such as brain, liver, or skeletal muscle (Supplementary Fig. 3). Nonetheless, we cannot rule out the possibility of CD45-Cre expression by very rare cells in other tissues or that non-haematopoietic cells might transiently activate CD45 expression during whatever nuclear reprogramming might occur after cell fusion.

CD45-Cre bone marrow cells were injected into four lethally a

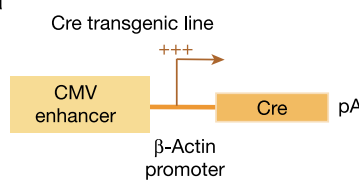

b

R26R transgenic line

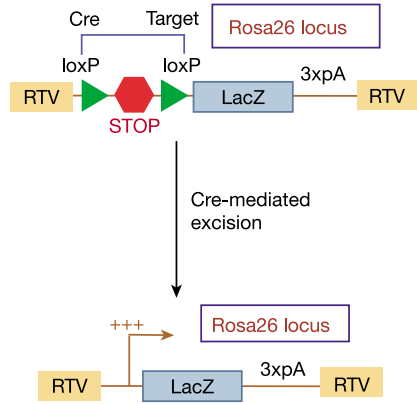

c

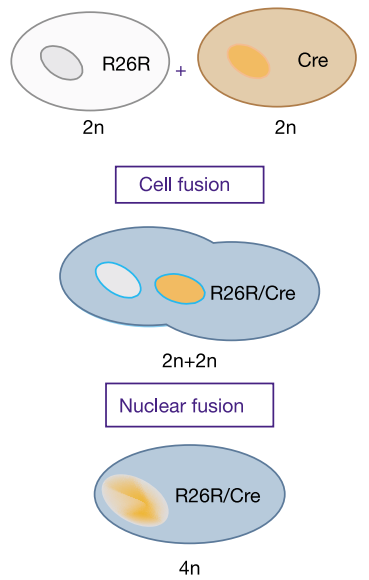

d

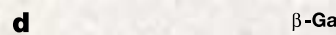

-Gal
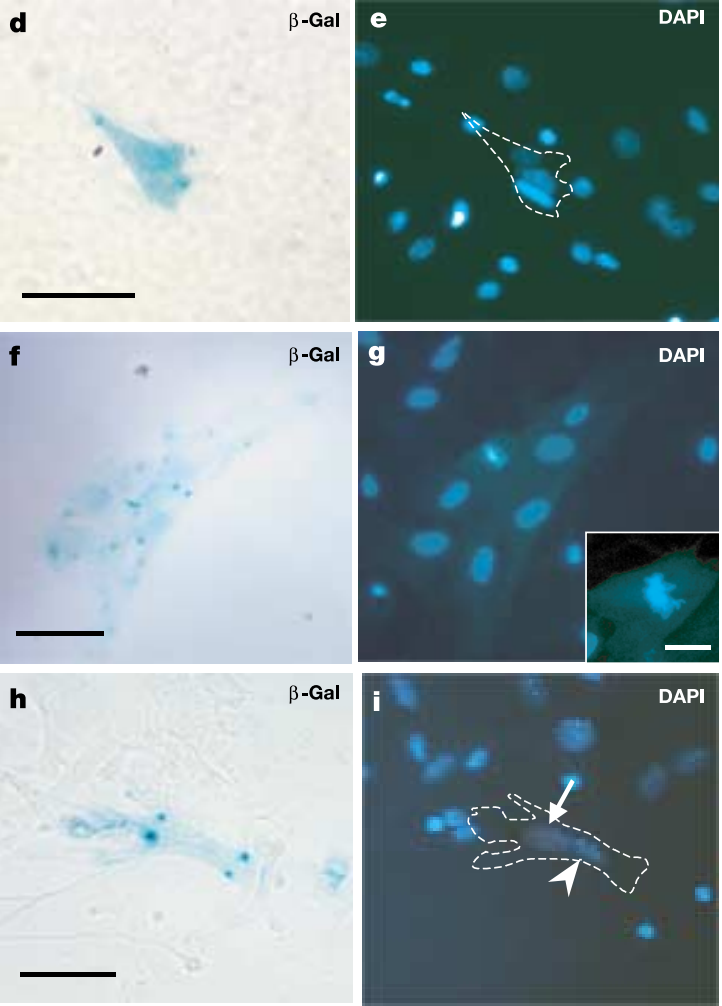

j.

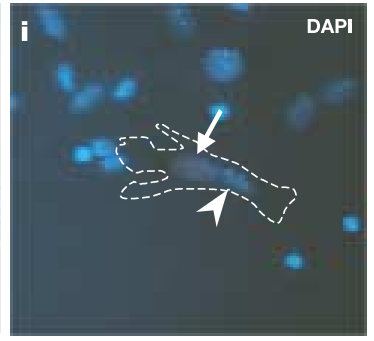

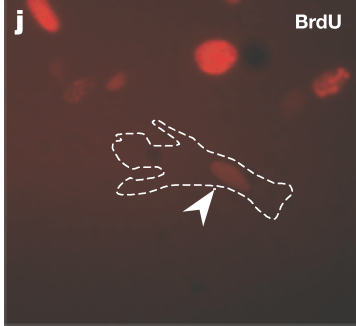

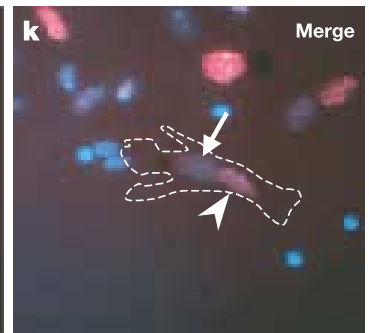

Figure 1 Method to detect cell fusion events. $\mathbf{a}, \mathbf{b}$, Schematic representation of the transgenes expressed by the mouse lines used. c, When a cell expressing Cre recombinase (a) fuses with a cell bearing the LacZ reporter transgene (b), the floxed stop cassette is excised and the LacZ reporter is expressed in the fused cell. LacZ expression can be detected by the generation of a blue precipitate after $\mathrm{X}$-gal staining. RTV, Integration retroviral sequence (LTR). $\mathbf{d}-\mathbf{g}$, Co-cultures of R26R BMSCs with $\mathrm{Cre}^{+}$ neurospheres stained for $\beta$-gal and the nuclear dye DAPI (4,6-diamidino-2-phenylindole). d, e, Multinucleated $\beta$-gal ${ }^{+}$fused cell (4 DIV). $\mathbf{f}, \mathbf{g}$, Colony of $\beta$-gal ${ }^{+}$fused cells (15 DIV). These cells were mononucleated and mitotically active, as demonstrated by a cell in metaphase (inset in $\mathbf{g}$ ). $\mathbf{h}-\mathbf{k}$, Co-culture of R26R BMSCs with BrdU-labelled $\mathrm{Cre}^{+}$ neurospheres. Bi-nucleated $\beta$-gal ${ }^{+}$fused cells (h) with one nucleus immunopositive for $\operatorname{BrdU}(\mathbf{j}, \mathbf{k}$, arrowhead) and the second nucleus negative for $\operatorname{BrdU}(\mathbf{i}, \mathbf{k}$, arrow). Scale bars: $\mathbf{d}, \mathbf{f}, \mathbf{h}, 20 \mu \mathrm{m} ; \mathbf{g}$ (inset), $5 \mu \mathrm{m}$. 


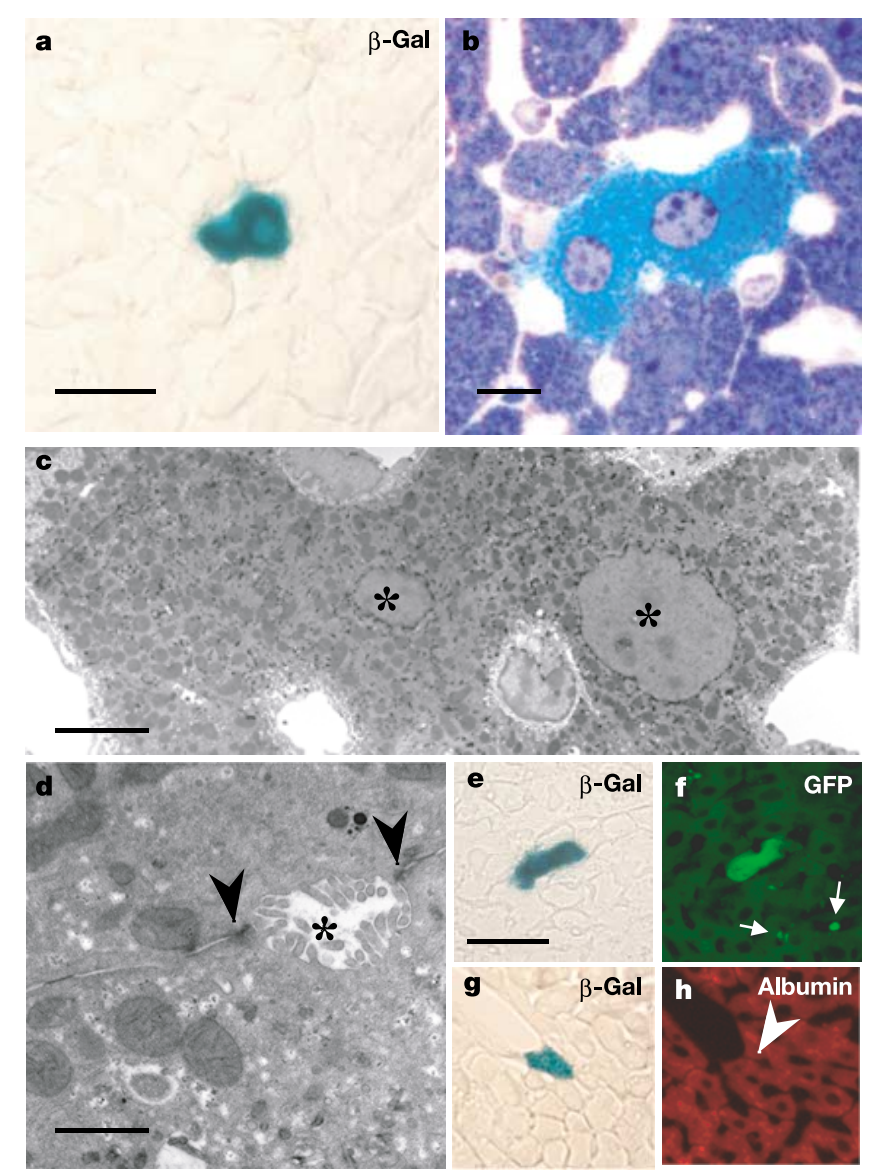

Figure 2 Fusion of hepatocytes with BMDCs after bone marrow transplantation. a, Representative image of a $\beta$-gal ${ }^{+}$hepatocyte detected in the liver of a R26R mouse transplanted with $\mathrm{GFP}^{+} / \mathrm{Cre}^{+}$bone marrow. b, c, Semi-thin section $(1.5 \mu \mathrm{m})$ of a $\beta$-gal ${ }^{+}$ hepatocyte (light blue) counter-stained with toluidine blue (b), and its morphology by electron microscopy (c). The hepatocyte contains two nuclei (asterisks), a mitochondriarich cytoplasm, and a very well developed endoplasmic reticulum typical of hepatocytes. d. High-magnification electron micrographs of a $\beta$-gal ${ }^{+}$hepatocyte containing glycogen storage granules, desmosomes (arrowheads) and bile canaliculi (asterisk). e, f, Ten-micrometre serial section showing a $\beta$-gal ${ }^{+}$hepatocyte $(\mathbf{e})$ and the adjacent section (f) exhibiting GFP expression. $\mathbf{g}, \mathbf{h}$, Serial sections showing a $\beta$-gal ${ }^{+}$hepatocyte (g) expressing the hepatocyte-specific marker albumin in the adjacent section (h). Scale bars: a, e, $50 \mu \mathrm{m} ; \mathbf{b}, 10 \mu \mathrm{m} ; \mathbf{c}, 5 \mu \mathrm{m} ; \mathbf{d}, 1 \mu \mathrm{m}$. irradiated R26R mice to look for fusion events (see 'Congenic bone marrow transplantation' section of Methods). The engrafted mice were analysed 10 months after transplantation. Consistent with the above observations, we found $\beta$-gal ${ }^{+}$hepatocytes in all four mice (Table 1; see also Supplementary Fig. 3e). $\beta$-gal ${ }^{+}$cardiomyocytes were found in two of the four mice, and $\beta-\mathrm{gal}^{+}$Purkinje cells were observed in one mouse (Table 1; see also Supplementary Fig. 3). As a negative control, we lethally irradiated seven R26R mice and transplanted them with $5 \times 10^{5}$ R26R bone marrow cells. No $\beta$-gal ${ }^{+}$cells were observed in these mice. These experiments suggest that haematopoietic cells fuse in vivo with cells in liver, heart and brain, but this does not rule out the possibility that other types of BMDCs might also participate in fusion.

In R26R mice that had been transplanted with bone marrow cells from $\beta$-actin-Cre-GFP mice, we looked for evidence of transdifferentiation in the grafted animals. $\mathrm{GFP}^{+}$cells that were negative for $\beta$-gal (that is, they did not fuse with recipient R26R cells) exhibited characteristics of microglia in the brain, of Kupffer or pit cells in the liver, and of macrophages in the heart (Supplementary Fig. 4). Each of these cell types are of haematopoietic origin ${ }^{20-22}$ and can fuse under certain conditions ${ }^{23}$, making them candidates for the haematopoietic cells that fused with resident cells. In contrast, no $\mathrm{GFP}^{+} / \beta$-gal ${ }^{-}$cells with the appearance of neural cells, hepatocytes, or cardiac muscle cells were observed. This suggests that cell fusion is the major mechanism by which haematopoietic cells can contribute to these tissues; however, our data do not rule out the possibility of rare transdifferentiation events, especially by other cell types or under other experimental conditions.

Our results suggest that BMDCs fuse with selective cell types in three organs. We did not observe evidence of fusion in skeletal muscle, gut, kidney, or lung in these experiments. The lack of evidence for fusion in these organs could be due to a lower efficiency of Cre-mediated recombination in these tissues or lower expression of the reporter gene (Supplementary Fig. 5). Alternatively, fusion may only occur in these tissues at a lower rate or under other experimental conditions, such as after injury.

With the exception of irradiation, the mice used in these experiments were healthy and did not have any pre-existing injury or pathology. Reconstitutions involving the $\beta$-actin-Cre-GFP mice were allogeneic and therefore could have experienced graft-versushost injury. However, reconstitutions involving CD45-Cre mice were congenic and did not involve any histoincompatibility. Although qualitatively similar results were observed in both contexts, considerable variation was observed from mouse to mouse in the extent to which fusion was observed. Therefore, the efficiency of somatic fusion in vivo is probably influenced by many variables.

Our results raise the fundamental question of whether fusion

\begin{tabular}{|c|c|c|c|c|c|c|c|}
\hline \multirow[b]{2}{*}{$\begin{array}{l}\text { Tissue and cell type } \\
\text { (number of cells analysed) }\end{array}$} & \multicolumn{3}{|c|}{2 months ${ }^{*}$} & \multicolumn{3}{|c|}{4 months $^{*}$} & \multirow{2}{*}{$\begin{array}{c}10 \text { monthst } \\
\text { (Fused) } \\
\beta-\text { gal }^{+}\end{array}$} \\
\hline & $\begin{array}{c}\text { (Transdifferentiated) } \\
\beta \text {-gal }{ }^{-} / \text {GFP }^{+}\end{array}$ & \multicolumn{2}{|c|}{$\beta$-gal $^{+} \begin{array}{c}\text { (Fused) } \\
\beta-\text { gal }^{+} / \mathrm{GFP}^{+}\end{array}$} & $\begin{array}{c}\text { (Transdifferentiated) } \\
\beta-\text { gal }^{-} / \text {GFP }^{+}\end{array}$ & \multicolumn{2}{|c|}{$\beta$-gal $^{+} \stackrel{\text { (Fused })}{\beta-\text { gal }^{+} / \mathrm{GFP}^{+}}$} & \\
\hline Liver/hepatocytes (20 sections, & $0,0,0$ & $21,11,9$ & $19,8,7$ & $0,0,0$ & $37,19,7$ & $9,5,2$ & $59,21,12$, \\
\hline Brain (cerebellum)/Purkinje cell $\quad\left(60\right.$ sections, $1.5 \times 10^{6}$ cells per mouse) & $0,0,0$ & $2,0,0$ & ND & $0,0,0$ & $5,3,1$ & ND & $5,0,0,0$ \\
\hline Heart/cardiomyocytes (25 sections, $\quad 7,750$ cells per mouse) & $0,0,0$ & $11,8,9$ & $9,5,6$ & $0,0,0$ & $18,7,2$ & $1,0,0$ & $71,3,0,0$ \\
\hline Other tissuesł & $0,0,0$ & $0,0,0$ & ND & $0,0,0$ & $0,0,0$ & ND & $0,0,0,0$ \\
\hline
\end{tabular}

Times indicate period after transplantation. Specimens were collected at different time points and were serially cut into 10- or 50- $5 \mathrm{~m}$ sections. Fused cells were detected by $\mathrm{X}$-gal staining. Alternating contiguous sections were screened for GFP fluorescence. $n=3$ ( 2 and 4 months) or 4 ( 10 months); each number represents the number of cells found in the sections analysed. The number of sections analysed per mouse and the approximate total number of cells scanned in those sections are indicated in the first column. ND, not determined.

*Allogeneic transplant of whole bone marrow from $\beta$-actin-Cre-GFP mice into R26R mice.

†Congenic transplant of whole bone marrow from CD45-Cre mice into R26R mice (both on C57BL background).

‡Gut, kidney, lung and skeletal muscle. 


\section{letters to nature}
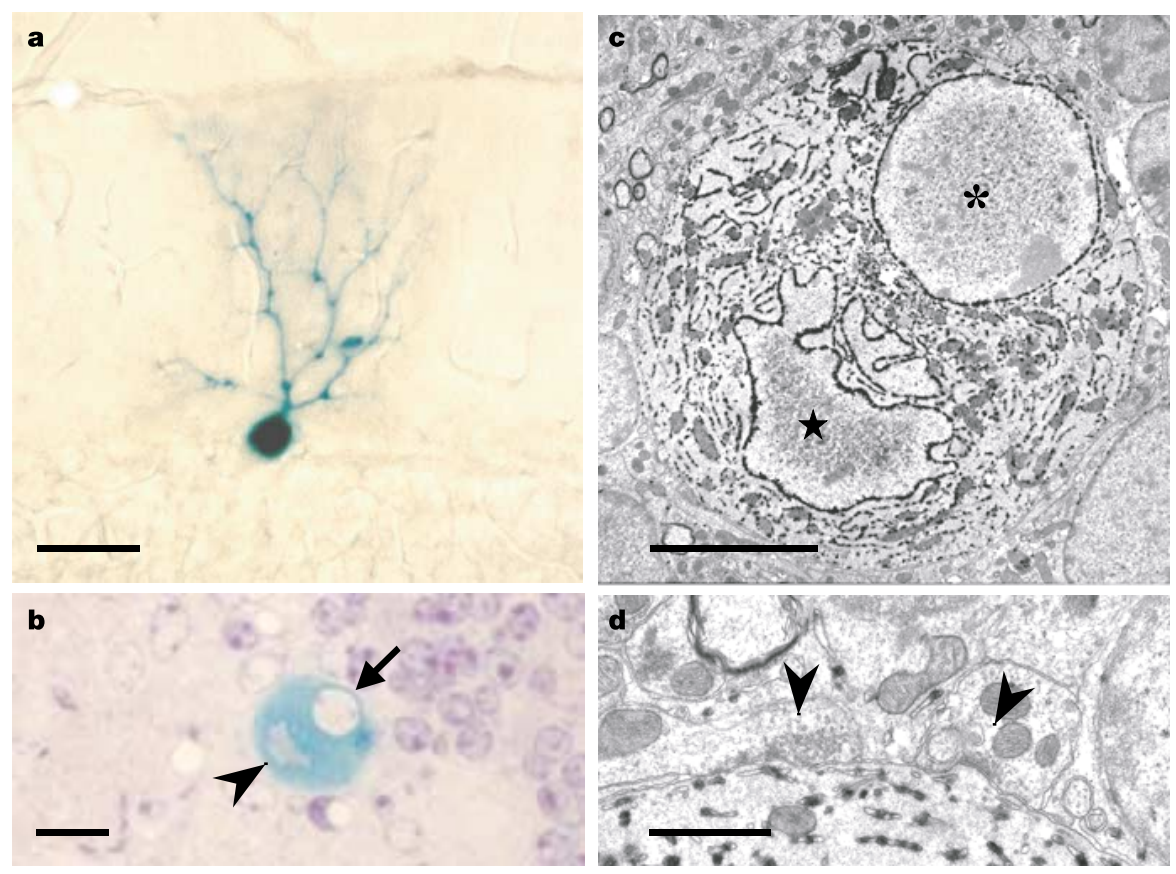

Figure 3 Purkinje cells fuse with BMDCs after bone marrow transplantation. $\mathbf{a}, \mathrm{A} \beta$-gal ${ }^{+}$ Purkinje neuron in the cerebellum of a R26R mouse transplanted with $\mathrm{Cre}^{+}$bone marrow cells. b, A 1.5- $\mu \mathrm{m}$-thick section of the $\beta$-gal ${ }^{+}$Purkinje cell soma (blue-green in colour) counter-stained with toluidine blue. This cell displays two nuclei (arrow and arrowhead) with very different morphological characteristics. c, Electron microscopy of the bi-nucleated fused Purkinje cell. Dark concretions in the reticulum and around the

nuclear membrane correspond to the X-gal precipitate. One nucleus is wrinkled and invaginated (star), typical of Purkinje cells, whereas the second nucleus (asterisk) has a homogeneous spherical shape, suggesting that the nuclei have different origins.

d, Electron microscopy photomicrograph of synaptic contacts (arrowheads) in a $\beta$-gal ${ }^{+}$ Purkinje cell. Scale bars: a, $40 \mu \mathrm{m} ; \mathbf{b}, 10 \mu \mathrm{m} ; \mathbf{c}, 5 \mu \mathrm{m} ; \mathbf{d}, 1 \mu \mathrm{m}$.

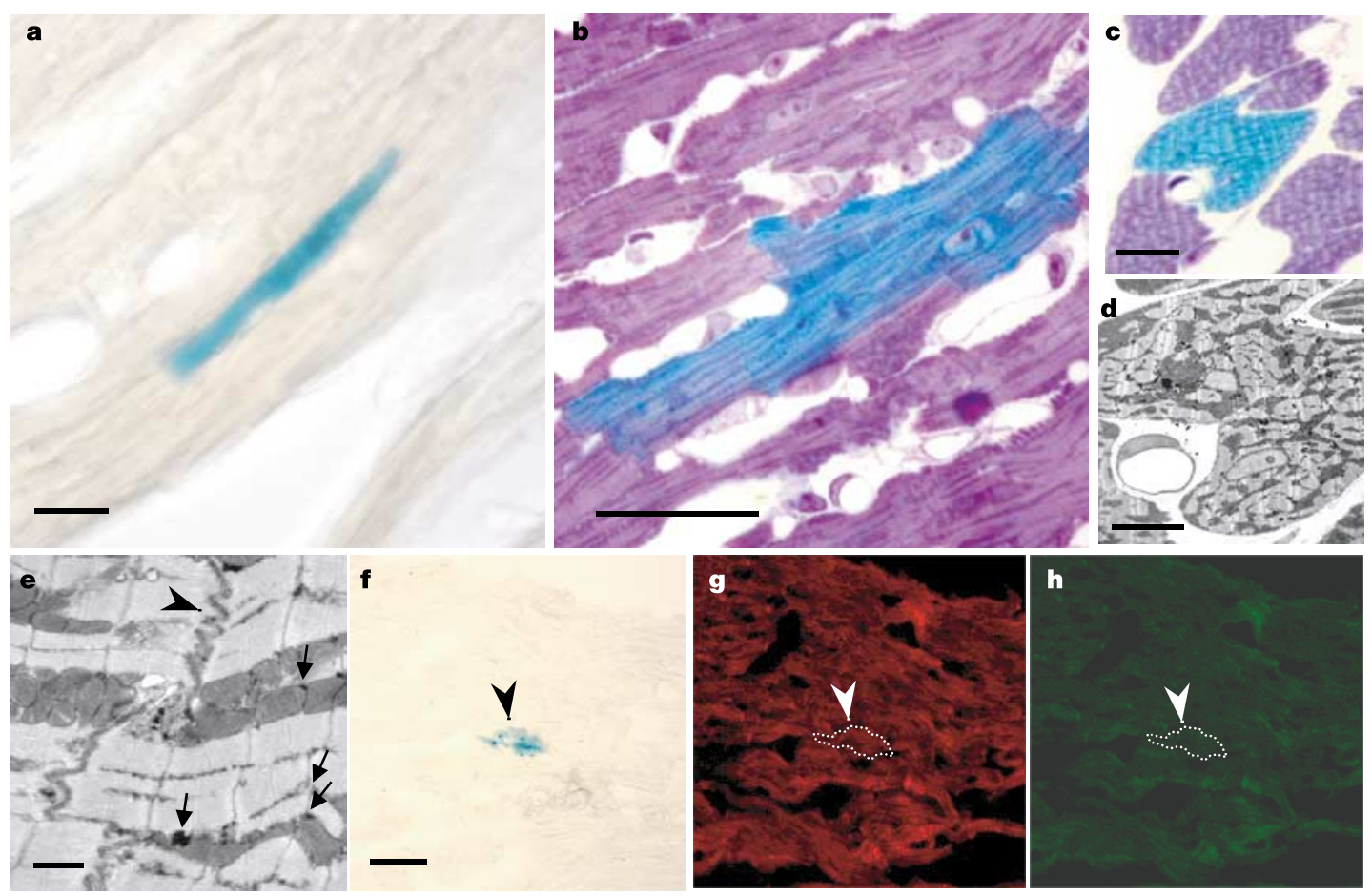

Figure 4 BMDCs fuse with cells in the heart. a-d, Sections at $50 \mu \mathrm{m}(\mathbf{a}), 1.5 \mu \mathrm{m}(\mathbf{b}, \mathbf{c})$ and electron microscopy (d) sections showing a $\beta$-gal ${ }^{+}$cardiomyocyte in a R26R mouse transplanted with $\beta$-actin-Cre-GFP-expressing bone marrow cells. Note that $\beta$-gal ${ }^{+}$ cardiomyocytes follow the orientation of other fibres. e, Electron microscopy photomicrograph showing mature structures of a $\beta$-gal ${ }^{+}$cardiomyocyte $(\beta$-gal precipitates, arrows), such as intercalated discs (arrowhead) and filament bands. $\mathbf{f}, \mathbf{g}$, A group of three $\beta$-gal ${ }^{+}$cardiomyocytes (f) were positive for cardiac troponin I (g) but negative for GFP (h). Scale bars: a, f, $25 \mu \mathrm{m} ; \mathbf{b}, 20 \mu \mathrm{m} ; \mathbf{c}, 10 \mu \mathrm{m} ; \mathbf{d}, 5 \mu \mathrm{m}$; e, $1 \mu \mathrm{m}$. 
between haematopoietic cells and cells of the brain, liver and heart has a physiological role in the development or maintenance of these organs. Interestingly, many hepatocytes and cardiomyocytes under normal conditions have two or more nuclei ${ }^{22,24}$. To our knowledge this is the first study to demonstrate Purkinje cells with two nuclei, but other studies have suggested that these neurons can be polyploid $^{25,26}$. Our results suggest that cell fusion may be the mechanism by which these cells become multinucleated or polyploid. Genetic material derived from blood cells may contribute through cell fusion to the survival and function of cells in different organs. Previous studies have shown that fused cells are positively selected during hepatic degeneration, helping to rescue a mutant mouse deficient for fumarylacetoacetate hydrolase ${ }^{8,9}$. Our observation that fusion is a major mechanism by which BMDCs contribute to the heart, liver and brain draws into question the rationale for clinical procedures based on the idea that transdifferentiation of BMDCs can lead to the de novo generation of heart or brain cells. Additional studies in animal models will be required to determine whether fusion by BMDC cells can be used in reparative cell therapy.

\section{Methods}

\section{Cell cultures}

Bone marrow cells from $\beta$-actin-Cre or R26R transgenic mice were collected by flushing tibias and femurs with RPMI medium 1640 (Gibco BRL) supplemented with 3\% fetal calf serum. Red blood cells were depleted using ice-cold ammonium chloride ( $140 \mathrm{mM}$ in Tris $17 \mathrm{mM}$ ), and bone marrow cells were plated at a density of $2-4 \times 10^{7}$ cells per $9.5 \mathrm{~cm}^{2}$ in Iscove's modified Dulbecco's medium (IMDM; Gibco BRL) supplemented with $10 \%$ fetal calf serum, $100 \mathrm{U} \mathrm{ml}^{-1}$ penicillin, $100 \mathrm{mg} \mathrm{ml}^{-1}$ streptomycin and $10 \mathrm{mg} \mathrm{ml}^{-1}$ glutamine (complete IMDM medium). The non-adherent cell population was removed after $48 \mathrm{~h}$ and the adherent BMDC layer washed once with fresh medium; cells were then continuously cultured for $1-4$ weeks.

For neurospheres, brain subventricular zone from 5-10-day-old $\beta$-actin-Cre or R26R mice was collected. After papain dissociation, neurospheres were cultured and expanded in the presence of both epidermal growth factor $\left(20 \mathrm{ng} \mathrm{ml}^{-1}\right.$; Peprotech) and fibroblast growth factor-2 (10 $\mathrm{ng} \mathrm{ml}^{-1}$; Peprotech), as described ${ }^{27}$. For BrdU labelling, neurospheres were cultured for $15 \mathrm{~min}$ in the presence of $2 \mu \mathrm{M} \mathrm{BrdU}$, washed, and expanded for two additional passages before being cultured with BMSCs. This procedure labelled $70-80 \%$ of the neurosphere cells. Fibroblasts were cultured as described previously ${ }^{28}$.

\section{Co-cultures}

BMDCs and dissociated neurospheres were mixed in a 1:1 ratio and plated on Matrigelcoated dishes (BD Bioscience) at a density of $2 \times 10^{5}$ cells $\mathrm{ml}^{-1}$ in complete IMDM medium. BMDCs and primary fibroblasts were cultured in a 1:1 ratio on plastic dishes in complete IMDM medium. After 4-15 days, co-cultures were washed and fixed in 2\% paraformaldehyde for $10 \mathrm{~min}$ and analysed by X-gal staining or immunohistochemistry. As a negative control, R26R BMDC monocultures were grown for 15 days in the presence of conditioned medium or cell extracts from Cre-expressing cells (data not shown). Cell extracts from Cre-expressing cells were freshly prepared by two freeze/thaw series and added to the culture medium.

\section{Animal care and bone marrow transplant}

Animal care and all procedures were approved by the Institutional Animal Care Committees at UCSF and the University of Michigan.

\section{Allogeneic bone marrow transplantation}

Homozygous mice expressing Cre recombinase under the control of the hybrid regulatory element CMV enhancer $\beta$-actin promoter ${ }^{11}$, and homozygous mice expressing GFP under the same promoter ${ }^{29}$ were bred to generate $\beta$-actin-Cre-GFP mice for use as bone marrow donors. Bone marrow cells from $8-10$-week-old Cre-GFP ${ }^{+}$mice were extracted as described, and 10-20 × $10^{6}$ cells were intraperitoneally injected into R26R mice irradiated with a single whole-body dose of 7.5 Gy. To avoid allograft rejection, mice that received the bone marrow transplantation procedure were treated one week before transplantation and 3 weeks after transplantation with Neoral cyclosporine $\left(100 \mathrm{mg} \mathrm{l}^{-1}\right.$; Novartis) in the drinking water. Drinking water was acidified and contained neomycin sulphate $\left(1 \mathrm{mgl}^{-1}\right.$; Sigma) to suppress pathogens.

\section{Congenic bone marrow transplantation}

In an independent experiment 8-10-week-old CD45-Cre 'knock-in' mice on a C57BL/KaThyl.1 background were used as donors of bone marrow cells. The generation of CD45Cre knock-in mice will be described elsewhere (E. Schaller and K.P., manuscript in preparation). Eight-week-old R26R mice on a C57BL/Ka-Thyl.2 background were used as recipients. Approximately $5 \times 10^{5}$ bone marrow cells were injected into the retro-orbital venous plexus of R26R mice lethally irradiated with two doses of 5.7 Gy each. The drinking water of the transplanted mice contained neomycin sulphate $\left(1 \mathrm{gl}^{-1}\right)$ and polymyxin $\mathrm{B}$ sulphate $\left(1 \times 10^{6} \mathrm{Ul}^{-1}\right)$ to suppress pathogens. On a monthly basis after transplantation, mice were bled and the peripheral blood was stained with antibodies against Thyl.1 and haematopoietic markers to confirm reconstitution.
CD45-Cre knock-in mice were bred with R26R to obtain CD45-Cre/R26R mice. The $\beta$-gal expression pattern in these mice was examined by X-gal staining of tissues and by fluorescein di- $\beta$-D-galactopyranoside (Molecular Probes) staining of haematopoietic cells. Bone marrow cells were incubated for $5 \mathrm{~min}$ at $37^{\circ} \mathrm{C}$ with $10 \mathrm{mM}$ fluorescein di- $\beta$-Dgalactopyranoside in a hypotonic solution ( $1: 1$ staining medium (HBSS plus $2 \%$ calf serum):distilled water). Haematopoietic stem cells $\left(\mathrm{Sca}_{-1}{ }^{+} \mathrm{c}^{-\mathrm{Kit}^{+}} \mathrm{Flk}^{+} 2^{-} \text {lineage }{ }^{-} \text {cells }\right)^{30}$ were analysed for $\beta$-gal expression using a FACS Vantage flow-cytometer (BectonDickinson) (Supplementary Fig. 3a).

\section{Tissue collection}

After 2, 4 or 10 months mice were anaesthetized and transcardially perfused with $0.9 \%$ saline followed by $50 \mathrm{ml} 4 \%$ paraformaldehyde or $2 \%$ paraformaldehyde plus $0.25 \%$ glutaraldehyde. Brain, spinal cord, liver, lung, kidney, heart, skeletal muscle and gut were dissected. Brain and one liver lobe were serially cut in $50-\mu \mathrm{m}$ vibrotome sections. The rest of the liver and other tissues were cryopreserved and frozen in optimum cutting temperature compound (Sakura-Finetec) at $-80^{\circ} \mathrm{C}$. Serial 10 - or $50-\mu \mathrm{m}$ sections were cut in a cryostat and stained with X-gal or by immunohistochemistry.

\section{X-gal staining and immunohistochemistry}

Specimens were placed in phosphate buffer containing $10 \mathrm{mM} \mathrm{K}_{3} \mathrm{Fe}(\mathrm{CN})_{6}$ and $10 \mathrm{mM}$ $\mathrm{K}_{4} \mathrm{Fe}(\mathrm{CN})_{6}$ along with the $\beta$-gal substrate X-gal $\left(1 \mathrm{mg} \mathrm{ml}^{-1}\right)$ (Molecular Probes) at $37^{\circ} \mathrm{C}$ for 8-12 h. Antibodies against albumin (A-6684; 1:100) and calbindin (C-9848; 1:1,000) were from Sigma, cardiac troponin I (sc-1881; 1:1,000) was from Santa Cruz Biotechnology, BrdU (M0744, 1:100) was from DAKO, CD45 (558750, 1:100) was from BD PharMingen, and Ibal was a gift from Y. Imai. Secondary antibodies anti-mouse-, goat- or rabbit-IgG $(\mathrm{H}+\mathrm{L})(\mathrm{Cy}-2,1: 400 ; \mathrm{Cy}-3,1: 400$; biotinylated, 1:500) conjugated were from Jackson Immunoresearch.

\section{Plastic embedding and electron microscopy}

Fifty-micrometre $\beta$-gal-stained sections were post-fixed with $1 \%$ osmium and $7 \%$ glucose for $2 \mathrm{~h}$, rinsed, dehydrated and embedded in araldite (Durcupan, Fluka). Semi-thin sections $(1.5 \mu \mathrm{m})$ were cut with a diamond knife and stained lightly with $1 \%$ toluidine blue. Semi-thin sections were re-embedded in an araldite block and detached from the glass slide by repeated freezing (liquid nitrogen) and thawing. Ultra-thin $(0.05 \mu \mathrm{m})$ sections were cut with a diamond knife, stained with lead citrate and examined under a Jeol100CX electron microscope.

Received 18 August; accepted 24 September 2003; doi:10.1038/nature02069. Published online 12 October 2003.

1. Morrison, S. J. Stem cell potential: can anything make anything? Curr. Biol. 11, R7-R9 (2001).

2. Orkin, S. H. \& Zon, L. I. Hematopoiesis and stem cells: plasticity versus developmental heterogeneity. Nature Immunol. 3, 323-328 (2002).

3. Krause, D. S. et al. Multi-organ, multi-lineage engraftment by a single bone marrow-derived stem cell. Cell 105, 369-377 (2001).

4. Orlic, D. et al. Bone marrow cells regenerate infarcted myocardium. Nature 410, 701-705 (2001).

5. Priller, J. et al. Neogenesis of cerebellar Purkinje neurons from gene-marked bone marrow cells in vivo. J. Cell Biol. 155, 733-738 (2001).

6. Terada, N. et al. Bone marrow cells adopt the phenotype of other cells by spontaneous cell fusion. Nature 416, 542-545 (2002).

7. Ying, Q. L., Nichols, J., Evans, E. P. \& Smith, A. G. Changing potency by spontaneous fusion. Nature 416, 545-548 (2002).

8. Vassilopoulos, G., Wang, P. R. \& Russell, D. W. Transplanted bone marrow regenerates liver by cell fusion. Nature 422, 901-904 (2003).

9. Wang, X. et al. Cell fusion is the principal source of bone-marrow-derived hepatocytes. Nature 422, 897-901 (2003).

10. Sauer, B. Inducible gene targeting in mice using the Cre/lox system. Methods 14, 381-392 (1998).

11. Lewandoski, M., Meyers, E. N. \& Martin, G. R. Analysis of Fgf8 gene function in vertebrate development. Cold Spring Harb. Symp. Quant. Biol. 62, 159-168 (1997).

12. Mao, X., Fujiwara, Y. \& Orkin, S. H. Improved reporter strain for monitoring Cre recombinasemediated DNA excisions in mice. Proc. Natl Acad. Sci. USA 96, 5037-5042 (1999).

13. Ianus, A., Holz, G. G., Theise, N. D. \& Hussain, M. A. In vivo derivation of glucose-competent pancreatic endocrine cells from bone marrow without evidence of cell fusion. J. Clin. Invest. 111, 843-850 (2003).

14. Weiss, S. et al. Multipotent CNS stem cells are present in the adult mammalian spinal cord and ventricular neuroaxis. J. Neurosci. 16, 7599-7609 (1996).

15. Palay, L. P. \& Chan-Palay, V. Cerebellar Cortex 15-25 (Springer, Berlin, 1974).

16. Weimann, J. M., Charlton, C. A., Brazelton, T. R., Hackman, R. C. \& Blau, H. M. Contribution of transplanted bone marrow cells to Purkinje neurons in human adult brains. Proc. Natl Acad. Sci. USA 100, 2088-2093 (2003).

17. Wagers, A. J., Sherwood, R. I., Christensen, J. L. \& Weissman, I. L. Little evidence for developmental plasticity of adult hematopoietic stem cells. Science 297, 2256-2259 (2002).

18. Ledbetter, J. A. \& Herzenberg, L. A. Xenogeneic monoclonal antibodies to mouse lymphoid differentiation antigens. Immunol. Rev. 47, 63-90 (1979).

19. van Ewijk, W., van Soest, P. L. \& van den Engh, G. J. Fluorescence analysis and anatomic distribution of mouse $\mathrm{T}$ lymphocyte subsets defined by monoclonal antibodies to the antigens Thy-1, Lyt-1, Lyt-2, and T-200. J. Immunol. 127, 2594-2604 (1981).

20. Ling, E. A. \& Wong, W. C. The origin and nature of ramified and amoeboid microglia: a historical review and current concepts. Glia 7, 9-18 (1993).

21. Gehrmann, J., Matsumoto, Y. \& Kreutzberg, G. W. Microglia: intrinsic immuneffector cell of the brain. Brain Res. Brain Res. Rev. 20, 269-287 (1995).

22. Arias, I. M., et al. The Liver Biology and Pathobiology (Lippincott Williams and Wilkins, Philadelphia, 2001)

23. Anderson, J. M. Multinucleated giant cells. Curr. Opin. Hematol. 7, 40-47 (2000).

24. Piper, H. M. \& Isenberg, I. Isolated Adult Cardiomyocytes (CRC, Boca Raton, 1989). 
25. Lapham, L. W. Tetraploid DNA content of Purkinje neurons of human cerebellar cortex. Science 159, 310-312 (1968)

26. Mares, V., Lodin, Z. \& Sacha, J. A cytochemical and autoradiographic study of nuclear DNA in mouse Purkinje cells. Brain Res. 53, 273-289 (1973).

27. Doetsch, F., Caille, I., Lim, D. A., Garcia-Verdugo, J. M. \& Alvarez-Buylla, A. Subventricular zone astrocytes are neural stem cells in the adult mammalian brain. Cell 97, 703-716 (1999).

28. Spector, D. L., Goldman, R. D. \& Leinwand, L. A. Cells: a Laboratory Manual 4.1-4.7 (Cold Spring Harbor Laboratory Press, New York, 1998).

29. Hadjantonakis, A. K., Gertsenstein, M., Ikawa, M., Okabe, M. \& Nagy, A. Generating green fluorescent mice by germline transmission of green fluorescent ES cells. Mech. Dev. 76, 79-90 (1998)

30. Christensen, J. L. \& Weissman, I. L. Flk-2 is a marker in hematopoietic stem cell differentiation: a simple method to isolate long-term stem cells. Proc. Natl Acad. Sci. USA 98, 14541-14546 (2001).

Supplementary Information accompanies the paper on www.nature.com/nature

Acknowledgements The authors thank G. Martin and P. Soriano for transgenic mouse lines, J. Maher at the UCSF Liver Centre for advice and assistance, and M. Kiel, O. Yilmaz and The University of Michigan Flow Cytometry Core for help with flow cytometry. R.P. thanks E. Schaller for technical help. M.A-D. thanks B. Rico, I. Cobos, T. Aragon and U. Borello for personal and scientific support. This work was supported by grants from NIH, the Sandler Foundation, the Spanish Ministry of Science and Technology (Ataxias Cerebelosa), and the Deutsche Forschungsgemeinschaft (DFG). R.P. was the recipient of a postdoctoral fellowship from the Spanish Ministry of Science and Technology.

Competing interests statement The authors declare that they have no competing financial interests

Correspondence and requests for materials should be addressed to A.A-B. (abuylla@itsa.ucsf.edu).

\section{SNARE-protein-mediated disease resistance at the plant cell wall}

\author{
Nicholas C. Collins ${ }^{1}$, Hans Thordal-Christensen ${ }^{2}$, Volker Lipka ${ }^{3}$, \\ Stephan Bau ${ }^{3}$, Erich Kombrink ${ }^{3}$, Jin-Long Qiu ${ }^{2}$, Ralph Hückelhoven ${ }^{4}$, \\ Mónica Stein ${ }^{5}$, Andreas Freialdenhoven ${ }^{3}$, Shauna C. Somerville \\ \& Paul Schulze-Lefert
}

${ }^{1}$ Sainsbury Laboratory John Innes Centre, Norwich, Norfolk NR4 7UH, UK ${ }^{2}$ Plant Research Department, Risø National Laboratory, DK-4000 Roskilde, Denmark

${ }^{3}$ Department of Plant Microbe Interactions, Max Planck Institute for Plant Breeding Research, Cologne D-50829, Germany

${ }^{4}$ Institute of Phytopathology and Applied Zoology Justus-Liebig-University, Giessen D-35392, Germany

${ }^{5}$ Department of Plant Biology, Carnegie Institute of Washington, Stanford, California 94305, USA

* These authors contributed equally to this work

Failure of pathogenic fungi to breach the plant cell wall constitutes a major component of immunity of non-host plant speciesspecies outside the pathogen host range-and accounts for a proportion of aborted infection attempts on 'susceptible' host plants (basal resistance) ${ }^{1-4}$. Neither form of penetration resistance is understood at the molecular level. We developed a screen for penetration (pen) mutants of Arabidopsis, which are disabled in non-host penetration resistance against barley powdery mildew, Blumeria graminis f. sp. hordei, and we isolated the PEN1 gene. We also isolated barley ROR2 (ref. 2), which is required for basal penetration resistance against $B$. g. hordei. The genes encode functionally homologous syntaxins, demonstrating a mechanistic link between non-host resistance and basal penetration resistance in monocotyledons and dicotyledons. We show that resistance in barley requires a SNAP-25 (synaptosome-associated protein, molecular mass $25 \mathrm{kDa}$ ) homologue capable of forming a binary SNAP receptor (SNARE) complex with ROR2. Genetic control of vesicle behaviour at penetration sites, and plasma membrane location of PEN1/ROR2, is consist- ent with a proposed involvement of SNARE-complex-mediated exocytosis and/or homotypic vesicle fusion events in resistance. Functions associated with SNARE-dependent penetration resistance are dispensable for immunity mediated by race-specific resistance $(R)$ genes, highlighting fundamental differences between these two resistance forms.

Most types of plant pathogens fail to produce disease on the majority of plant species. Although 'non-host' resistance is the most common form of resistance, its basis is poorly understood owing to the dearth of tractable genetic systems. This contrasts with 'racespecific' resistance triggered by corresponding AVIRULENCE $(A V R) / R$ genes in otherwise compatible host-pathogen interactions, for which many components have been identified ${ }^{5}$. Suicide of cells surrounding the infection site (often referred to as the hypersensitive response) typically accompanies $R$-gene-mediated resistance, and hypersensitive-response-like cell death can also be associated with non-host resistance. These drastic measures form secondary lines of defence that are normally triggered once a fungus has overcome active defences at the plant cell periphery ${ }^{3,6}$.

We investigated whether the immunity of the model plant Arabidopsis to the barley powdery mildew B. g. hordei could be used to develop a system for dissecting non-host resistance. Blumeria g. hordei conidiospores germinated on Arabidopsis but most sporelings failed to enter the plant cells, accompanied by the formation of a cell wall deposition (papilla) by the plant cell directly beneath penetration attempts. About $10 \%$ of sites showed successful penetration as indicated by the presence of a fungal feeding structure (haustorium; Fig. 1a); however, most of the penetrated cells underwent hypersensitive-response-like cell death (Fig. 1b), manifested as whole-cell autofluorescence. Haustoria became encased in deposits containing callose, as revealed by aniline blue staining. Rarely, short hyphae were produced on the leaf surface (Fig. 1a), indicative of successful nutrient uptake through haustoria, before further fungal growth was invariably halted. Independent screens for Arabidopsis mutants allowing increased penetration by B. g. hordei (pen mutants) were performed, using either whole-cell autofluorescence or induced callose deposition as indicators of penetration. Mutants were identified for at least three genes (PEN1, -2 and -3; data not shown). Mutant alleles of PEN1 were recovered from each screen.

Map-based cloning of PEN1, supported by the sequencing of four mutant alleles (Fig. 1c), revealed that it encodes $A$. thaliana syntaxin (At)SYP121 (ref. 7). The pen1-1 mutation results in a stop codon early in the open reading frame and presumably leads to complete loss of PEN1 function. pen1-1 mutant plants allowed a sevenfold higher incidence of $B$. g. hordei penetration compared with wildtype plants, as well as a concomitant increase in the incidence of hypersensitive-response-like cell death induced by B. g. hordei (Fig. 1b). Further B. g. hordei growth was invariably arrested in pen1-1 plants. Thus, although impairment of penetration resistance would be necessary for Arabidopsis to be an effective host for B. g. hordei, it is not sufficient. Nicotiana tabacum SYR1, a tobacco homologue of PEN1/AtSYP121 (AtSYR1), has been suggested to have roles in mediating abscisic acid signalling, stomatal closing and normal growth in tobacco ${ }^{8}$; however, pen 1 mutants showed no discernible defects in general growth, stomatal closing ability, or root development (data not shown).

The barley-B. g. hordei combination also provides a useful system for the analysis of penetration resistance. Mutants of the barley $M L O$ suppressor of resistance show highly effective penetration resistance against all tested $B$. g. hordei isolates. ROR1 and ROR2 were identified in a mutant search as genes required for full $\mathrm{mlo}$ resistance ${ }^{2}$, but they also contribute to low-level basal penetration resistance expressed in 'susceptible' wild-type $M L O$ backgrounds (Supplementary Fig. 1a). Combining mutations in ROR1 and ROR2 had an additive effect on susceptibility (Supplementary Fig. 1b). We isolated ROR2 using a barley-rice syntenic-map-based cloning 